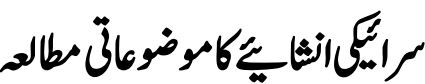

\section{Thematic Study of Seraiki Inshaiyah}

Muhammad Ramzan*

Dr. Syed Safdar Hussain**

\section{Abstract}

This paper is primarily dealing with the Saraiki light essay and its concerened subjects. The paper explained that society has different mode of literature to express it through poetry or light essay during every era of life according to the regional circumstances. Saraiki region which is culturaly rich in literature. The have great cultural heritage, people here expressed and shared their views through different subjects of light essays. The Saraiki light essay is also considered an important source of socio-cultural and religious aspects i.e. life, recreation, nature, traditions, entertainment and other life concerned issues. Saraiki light essay is full of humer, sincerety and love of humanity which are the important topics of the Saraiki society in Saraiki light essay. This research paper is an attempt to explore all these topics of the Saraiki society which used in Saraiki light essay.

Keywords: Saraiki, Society, Literature, Light Esay, Social, Culture, Traditions,

\section{موضوعكاتحارف}

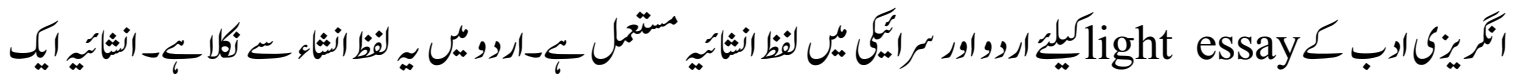

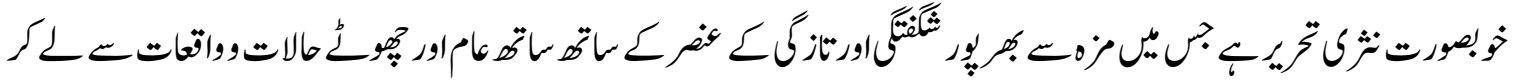

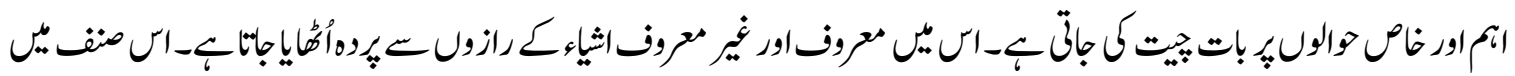

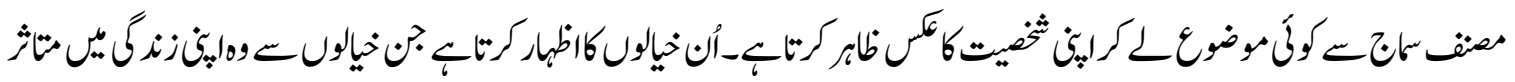

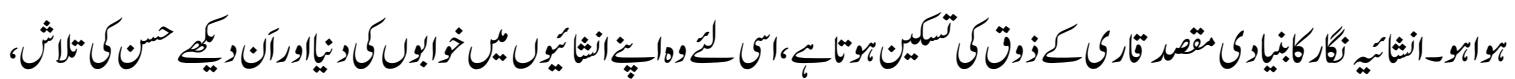

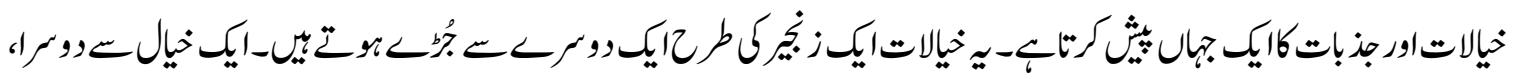

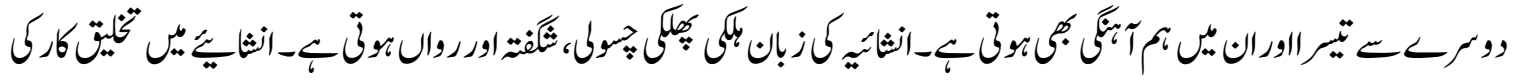

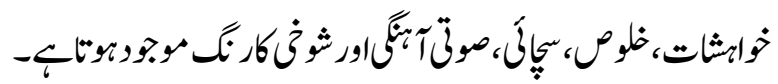
موضور6C

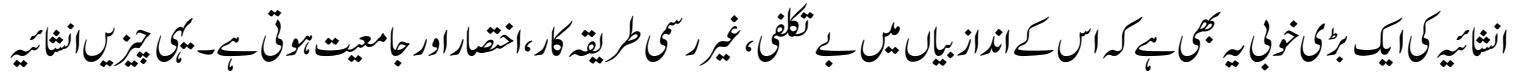

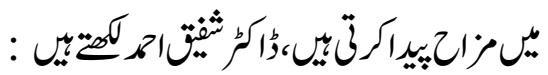

${ }^{*}$ Ph. D Scholar, Department of Saraiki, IUB, Bahawalpur.

Email: mrchohan1978@gmail.com

${ }^{* *}$ Associate Professor, Saraiki Department of Saraiki, IUB, Bahawalpur.

Email: ssafdariub@gmail.com 


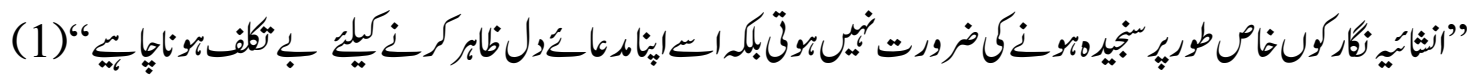

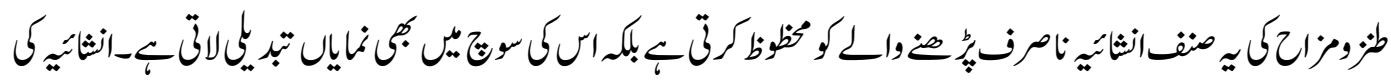

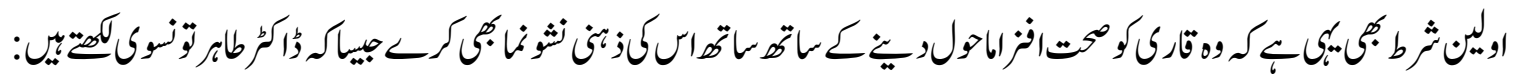

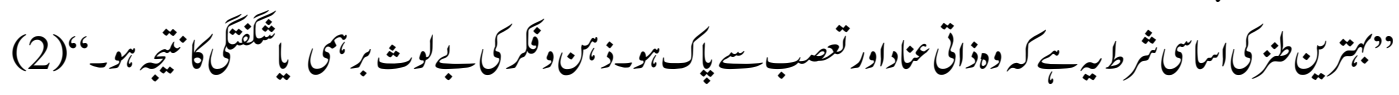

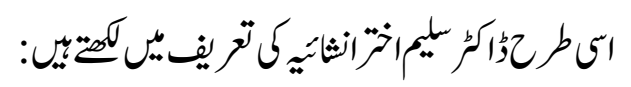

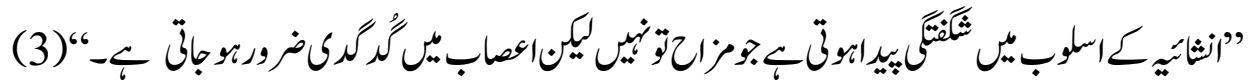

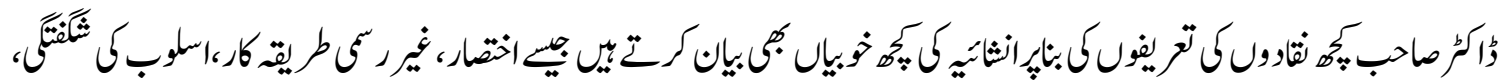

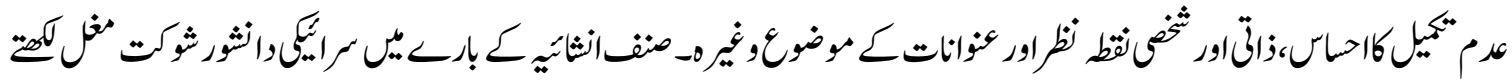

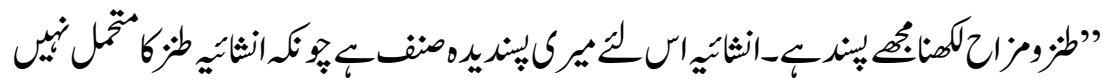
:

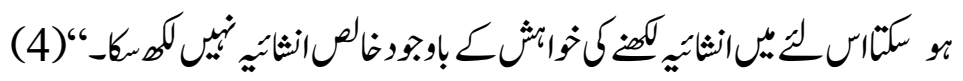

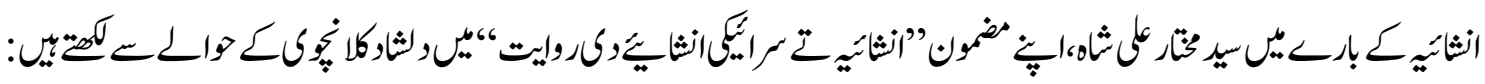

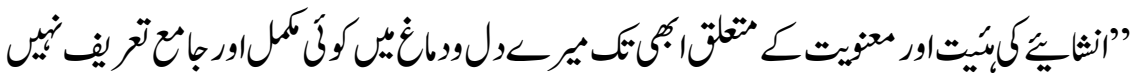

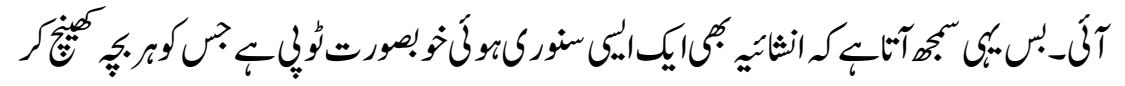

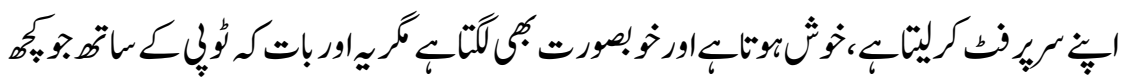

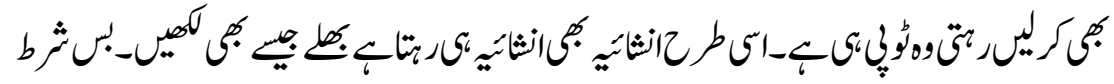

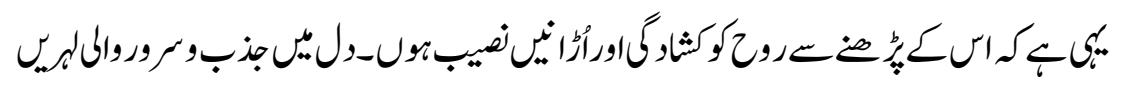

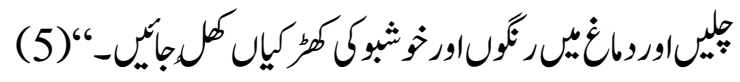

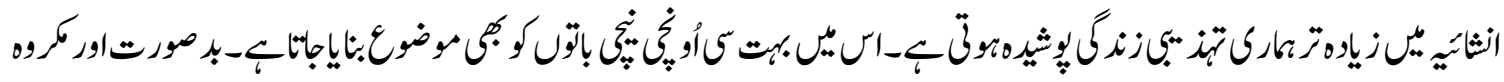

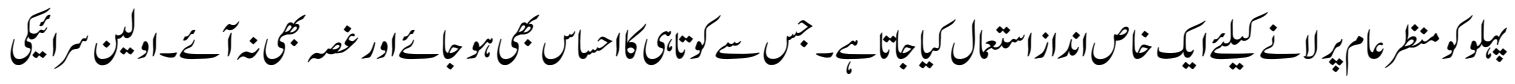

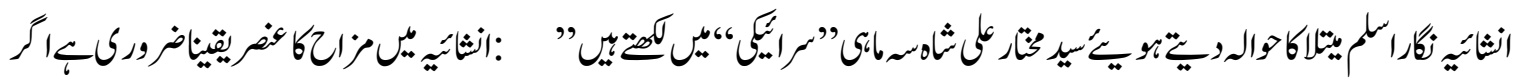

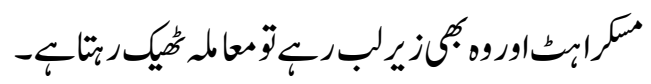

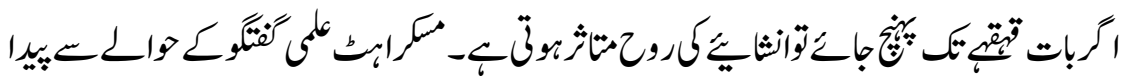

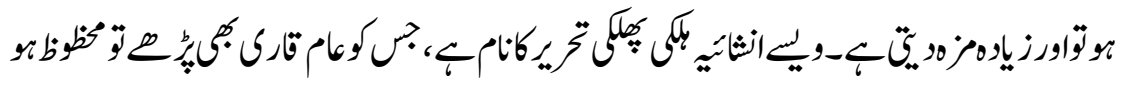




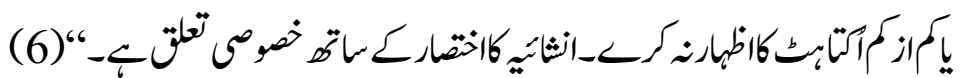

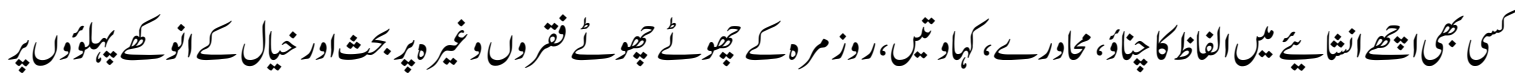

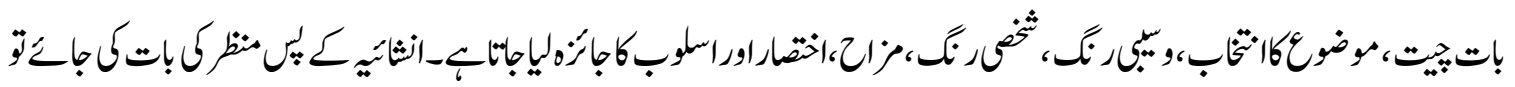

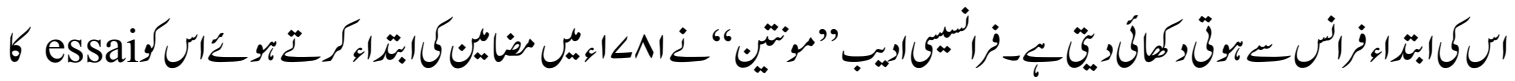

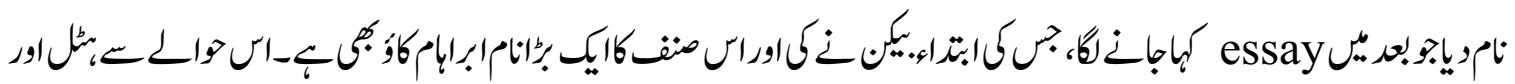

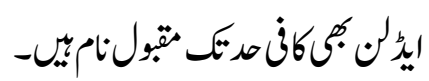

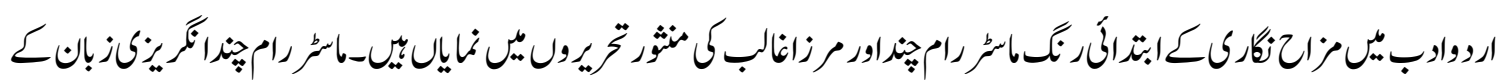

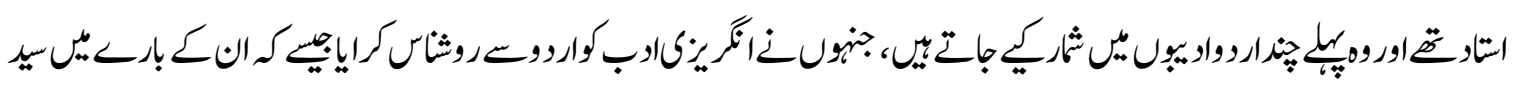

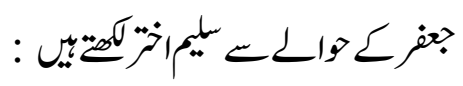

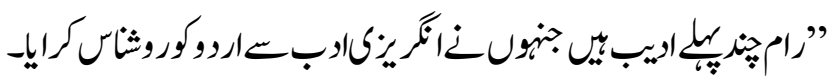

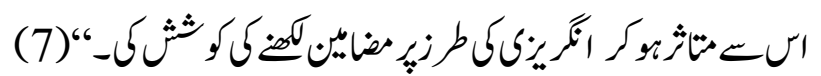

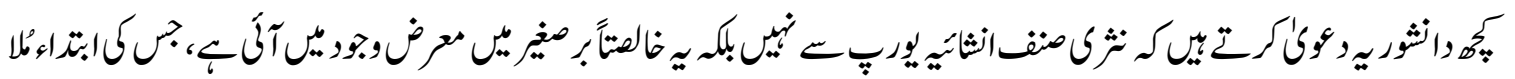

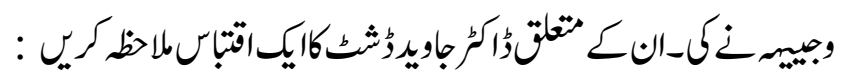

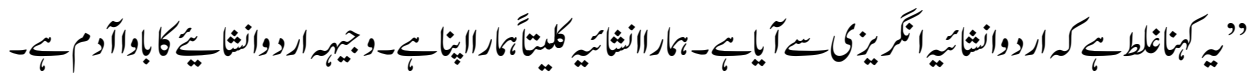

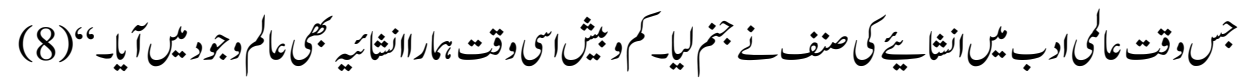

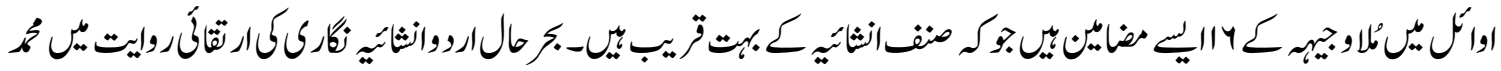

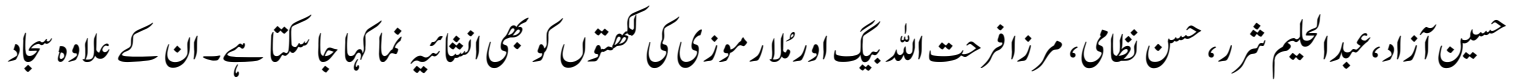

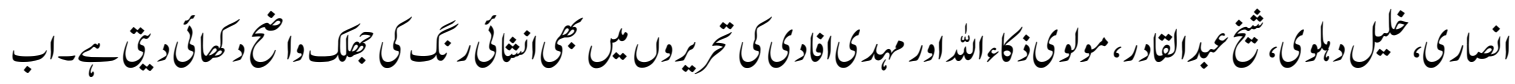

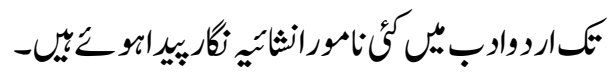

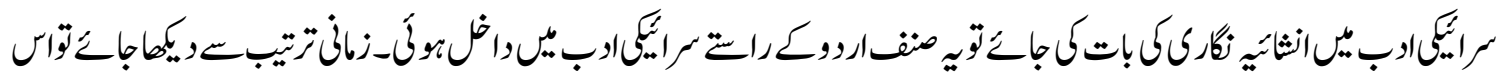

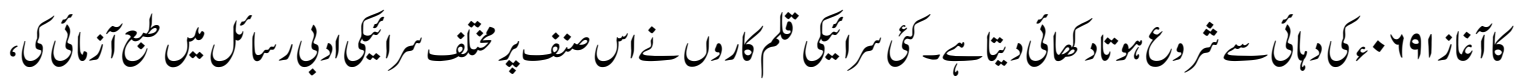

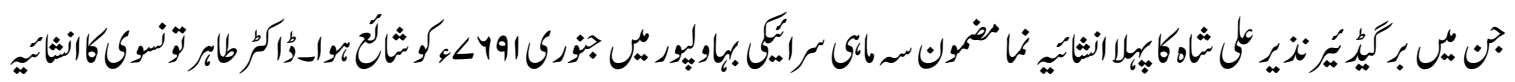

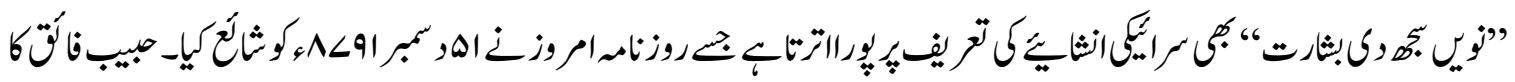

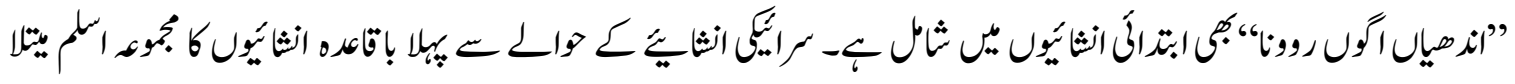




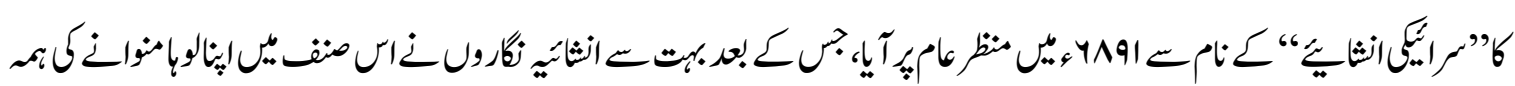

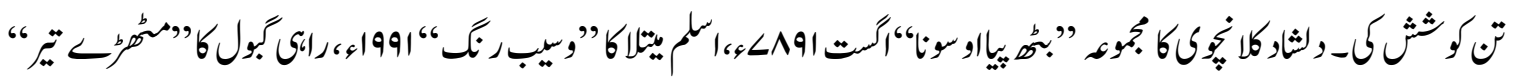

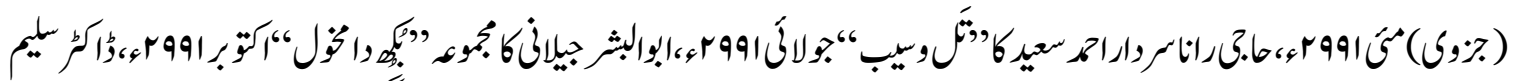

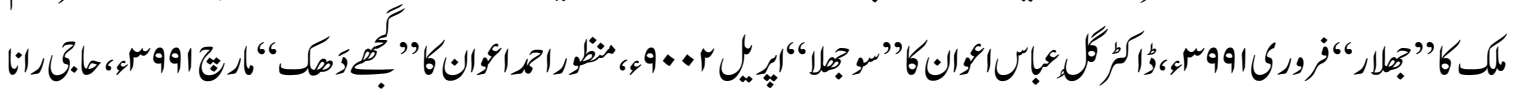

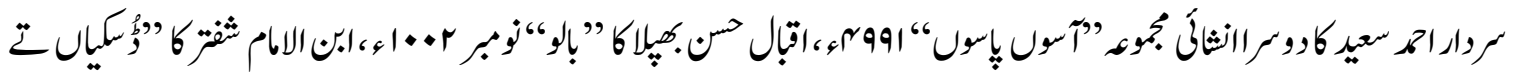

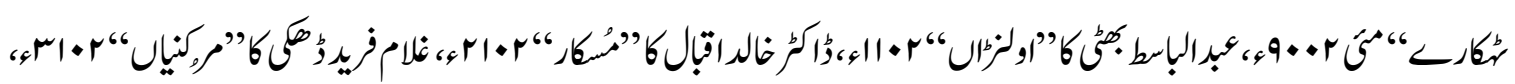

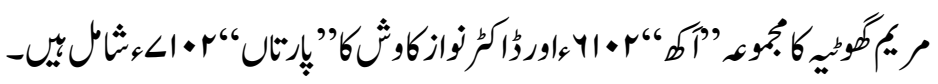

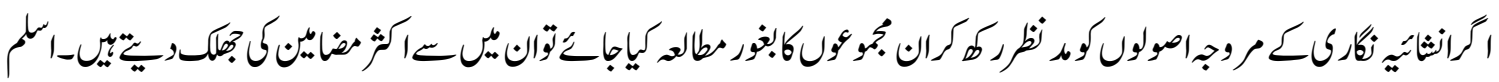

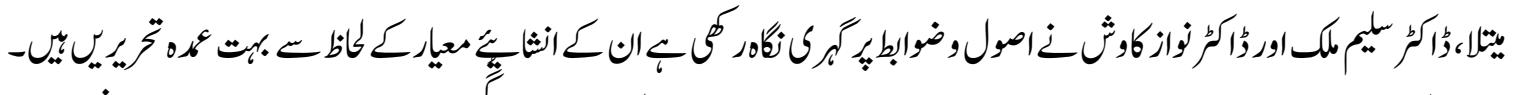

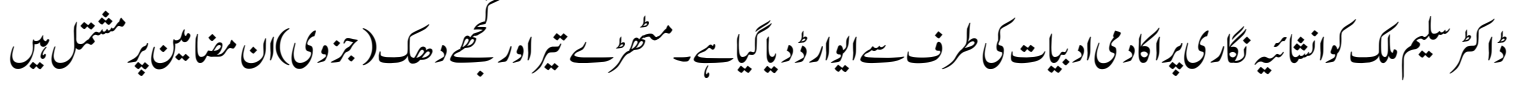

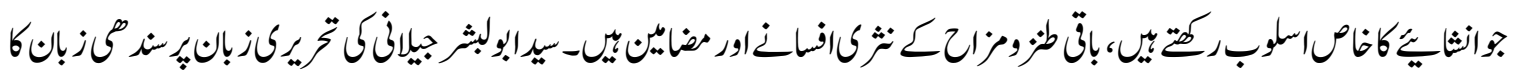

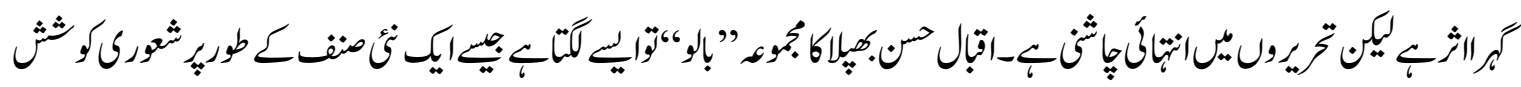

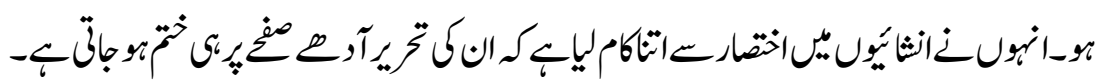

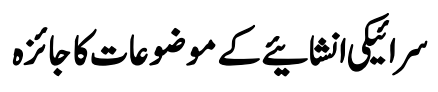

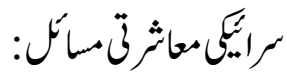

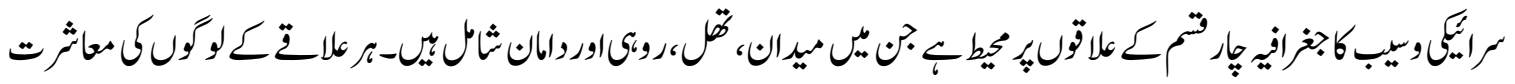

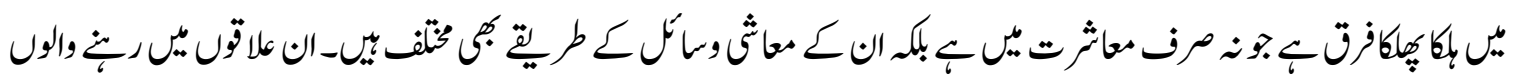

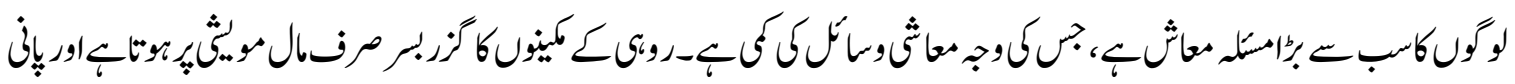

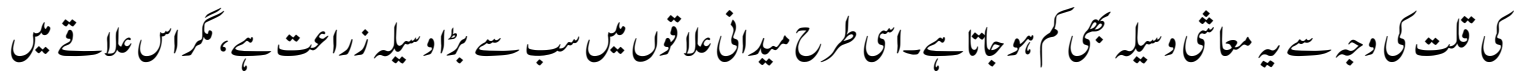

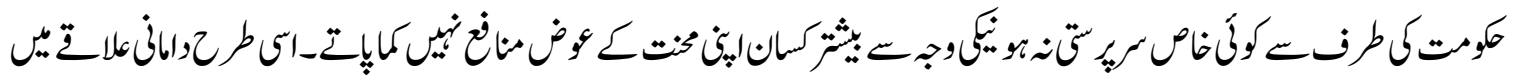

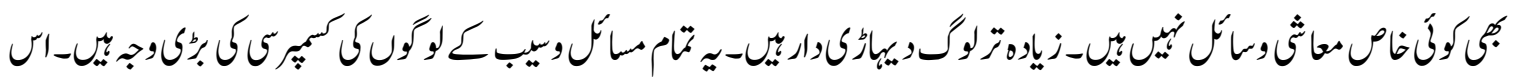

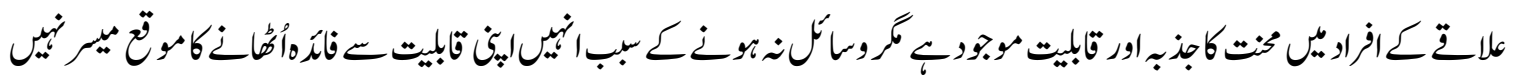

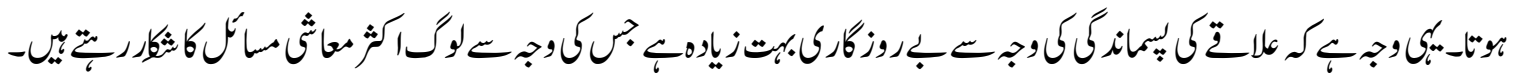

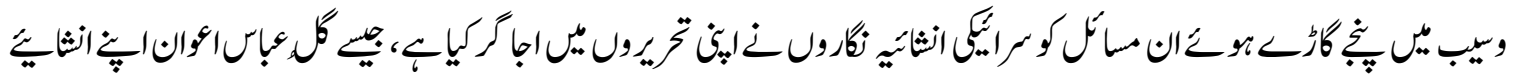




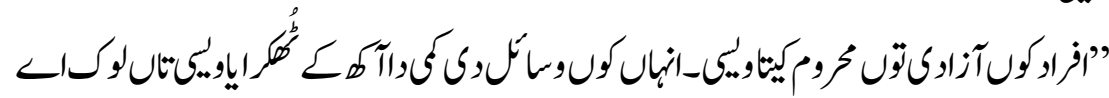

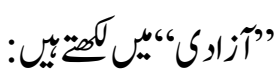

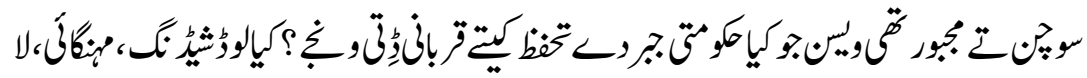

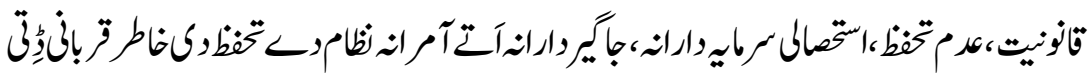

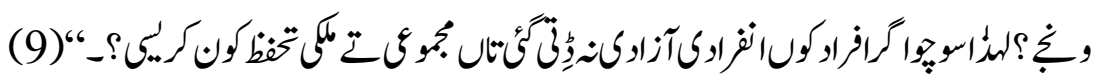

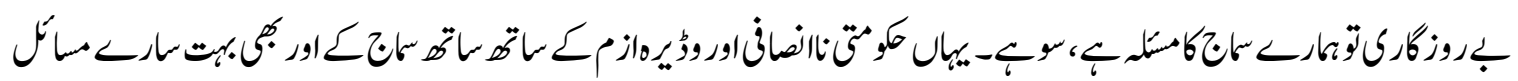

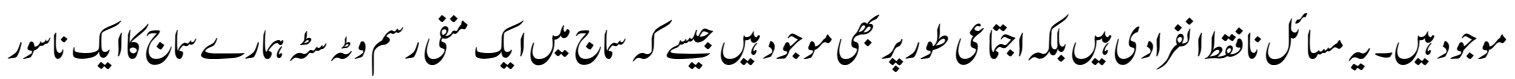

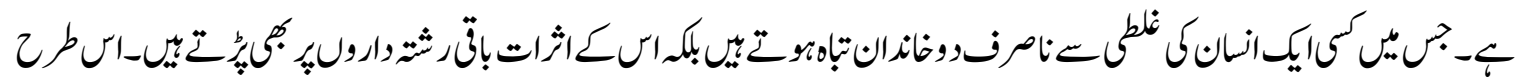

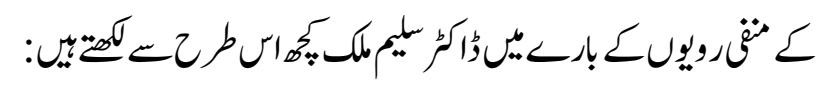

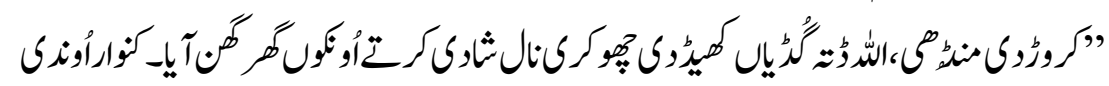

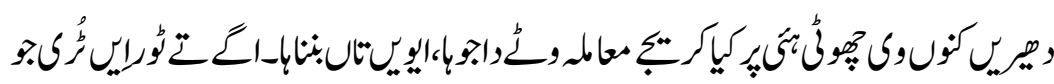

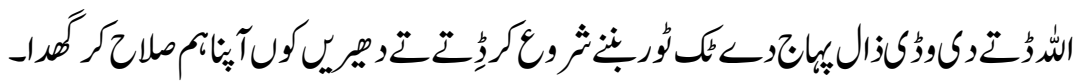

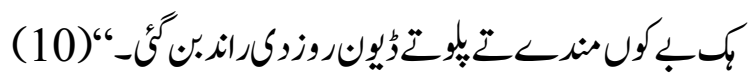

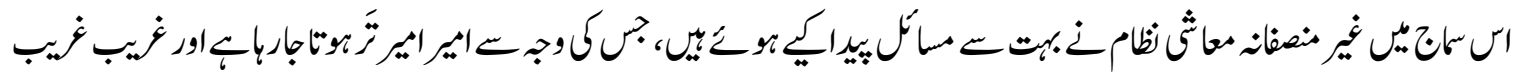

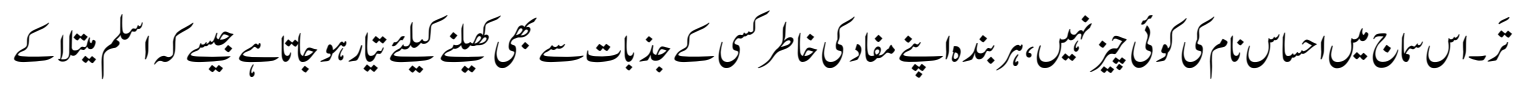

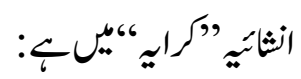

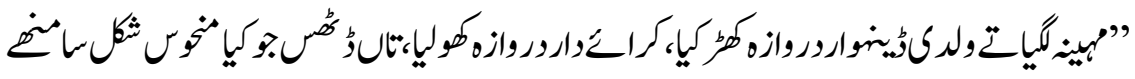

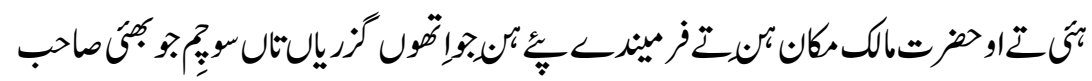

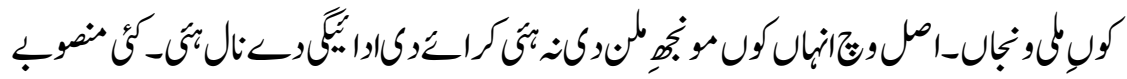

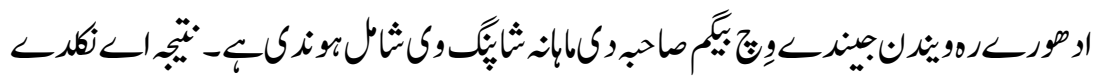

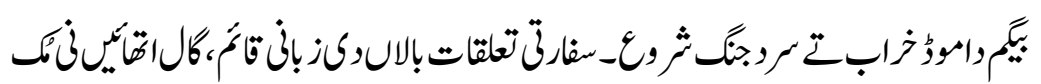

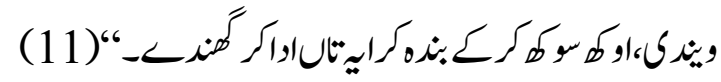

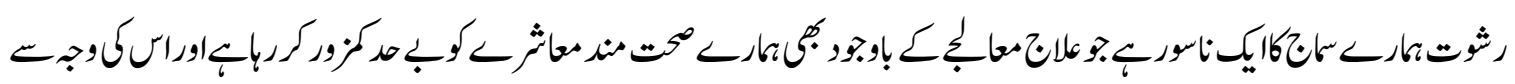

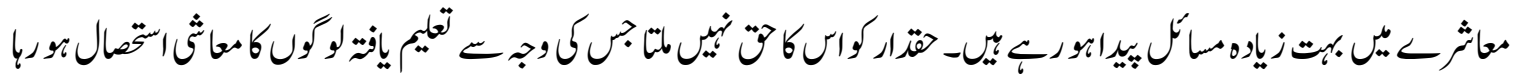




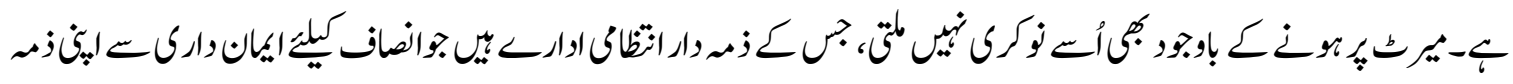

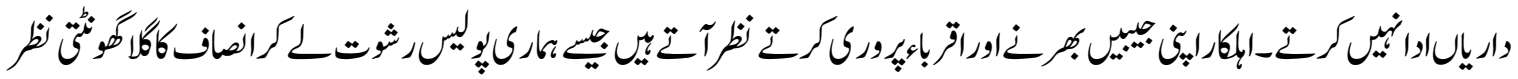

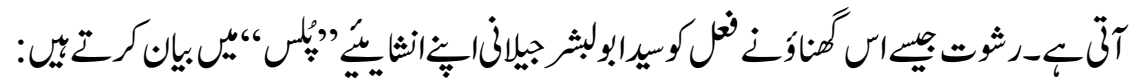

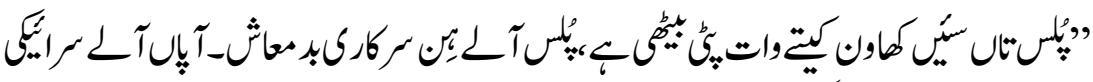

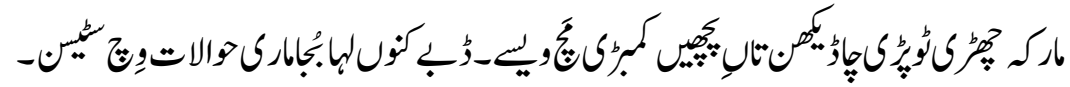

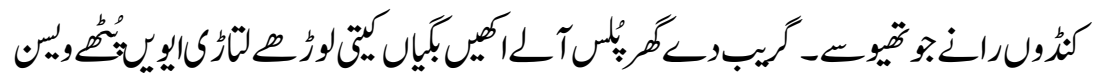

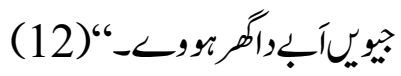

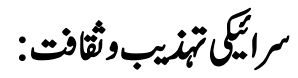

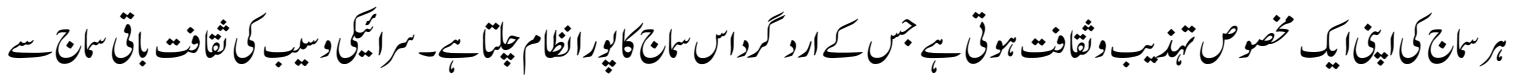

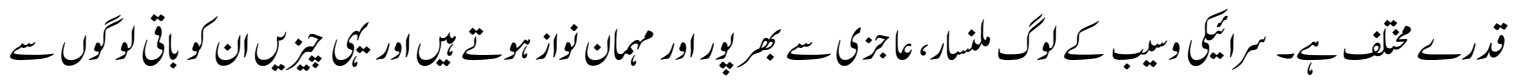

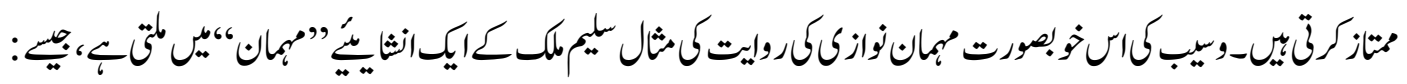

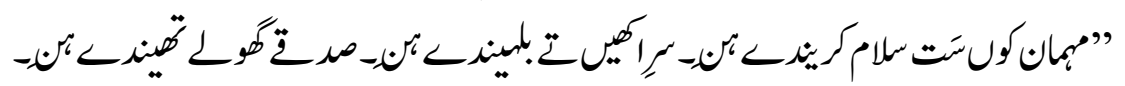

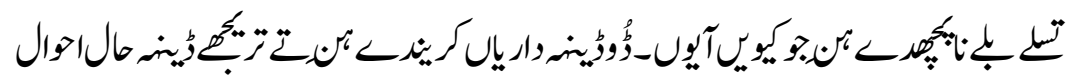

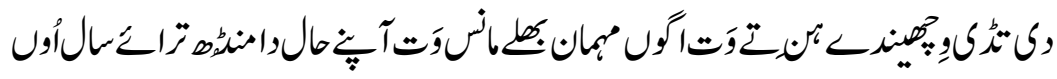

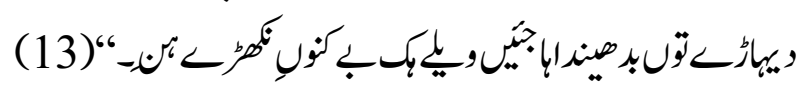

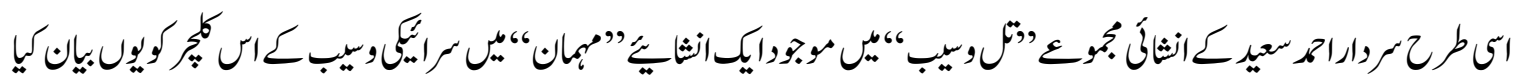

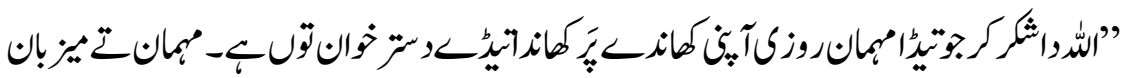

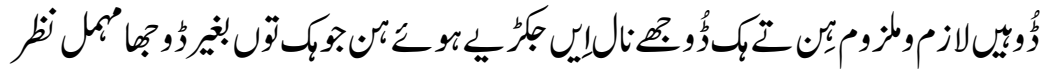

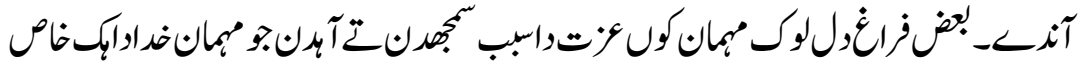

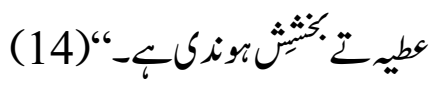

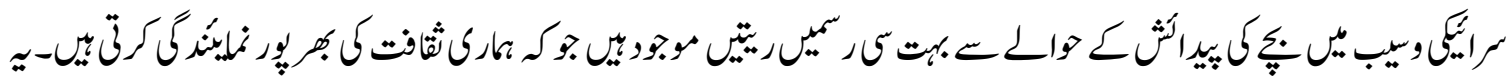

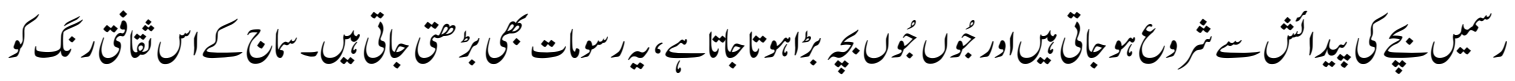

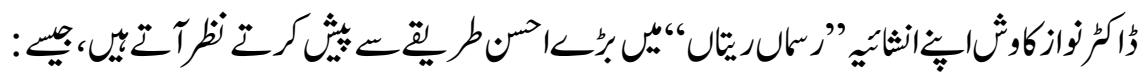




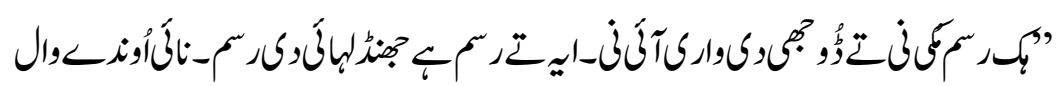

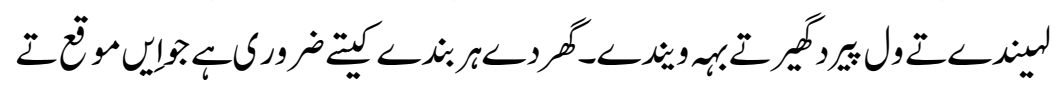

(15)“国

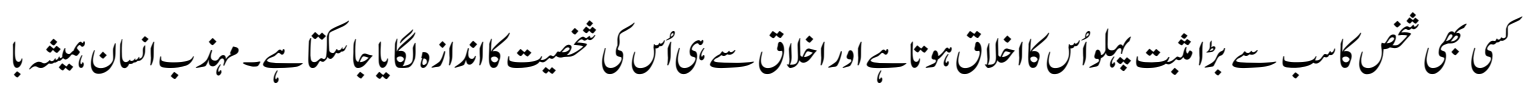

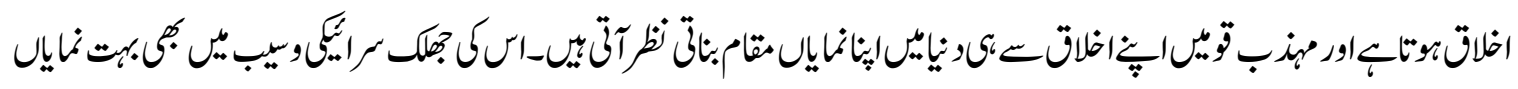

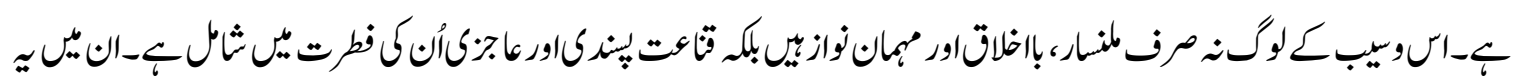

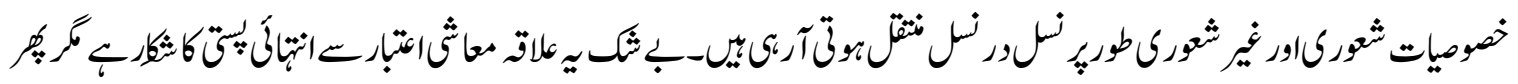

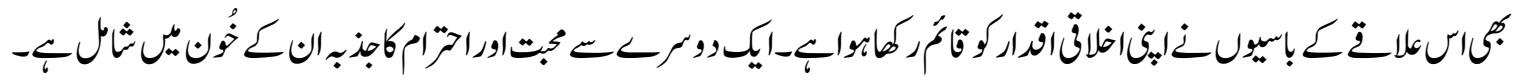

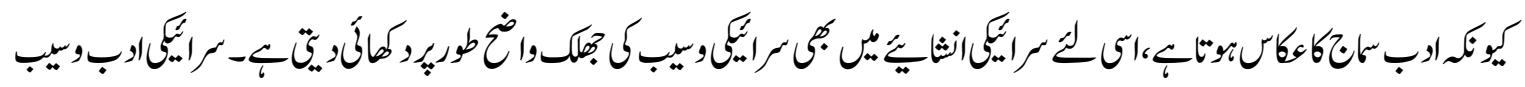

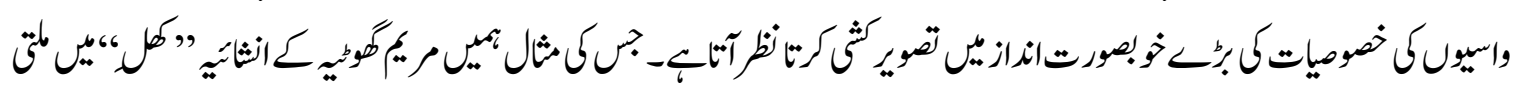

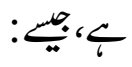

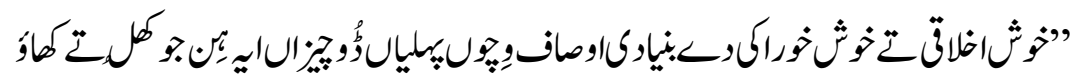

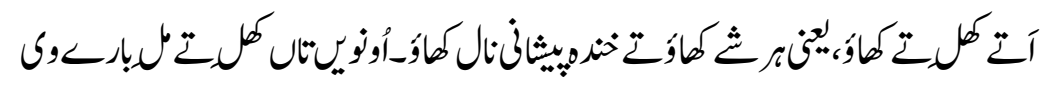

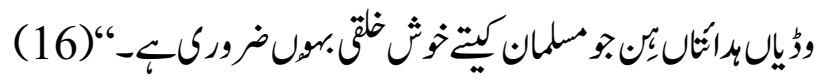

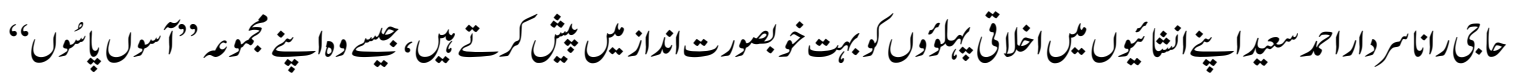

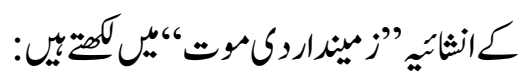

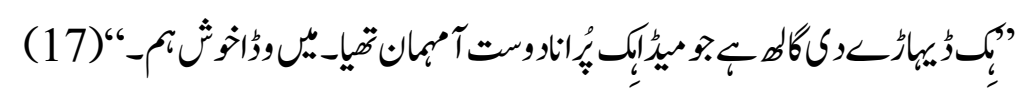

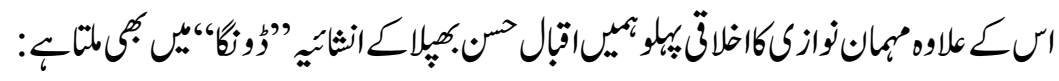

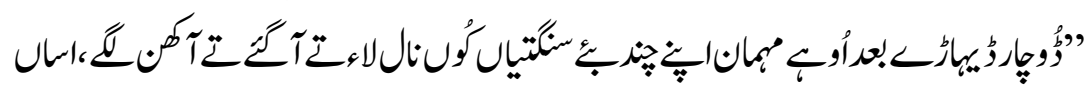

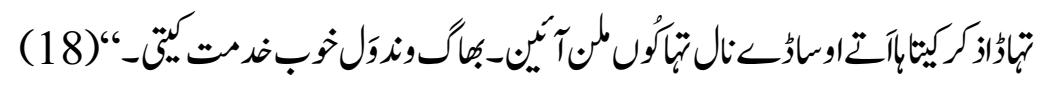

ريكانماثرت:

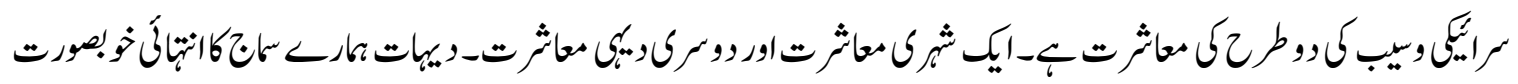

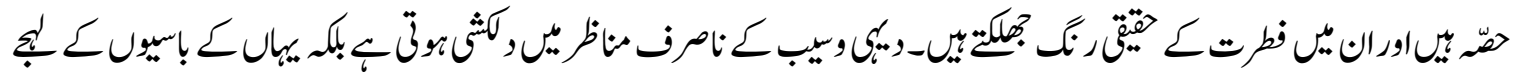




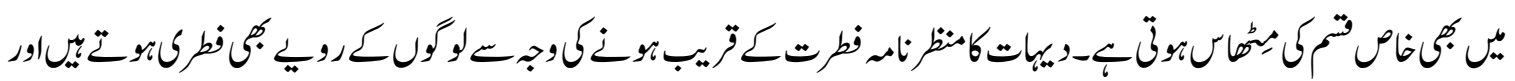

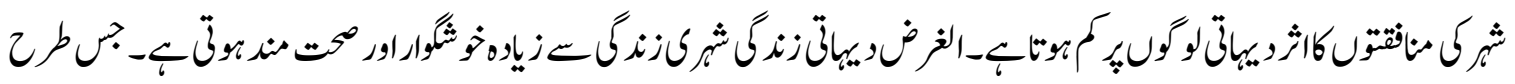

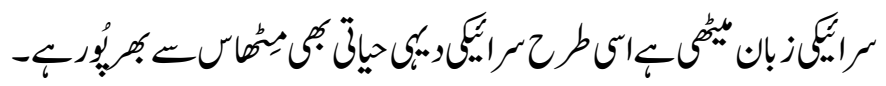

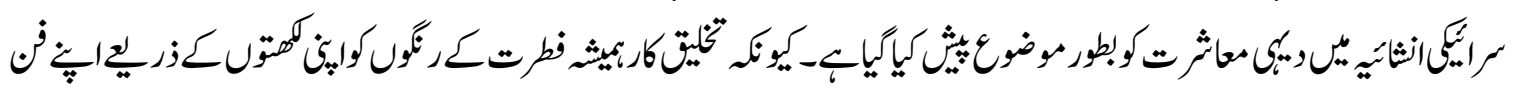

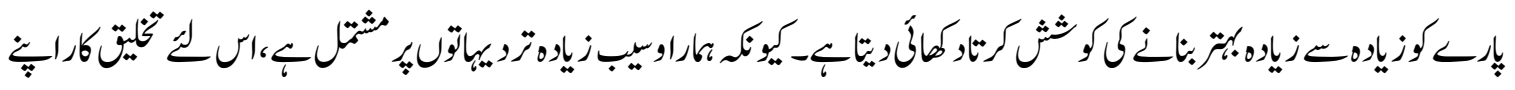

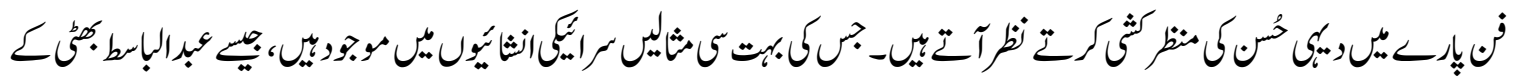

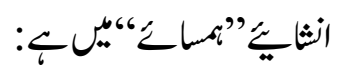

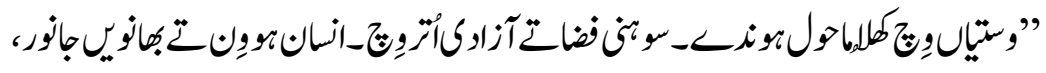

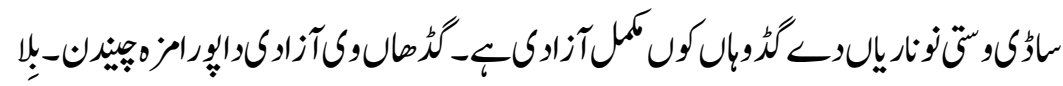

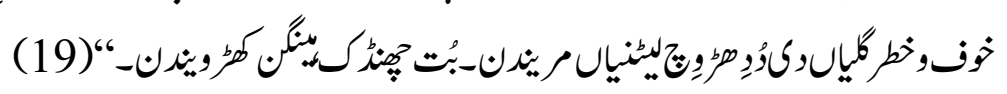

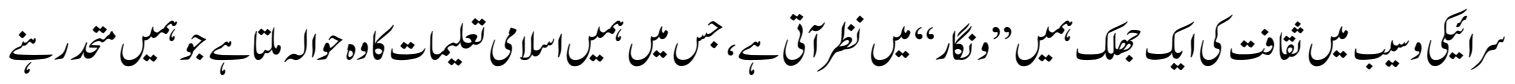

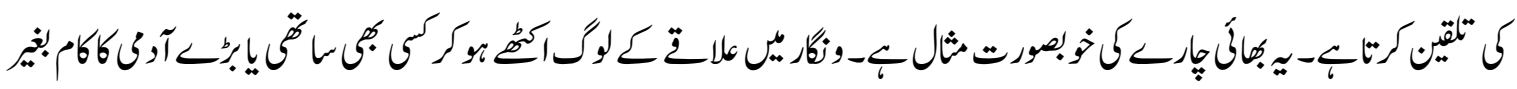

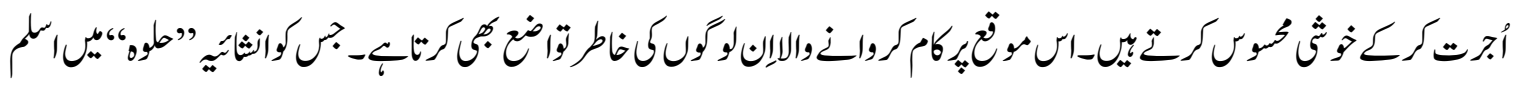

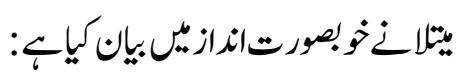

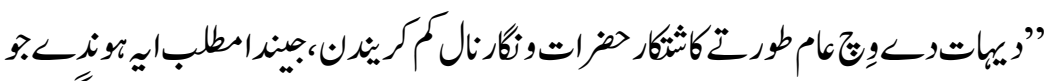

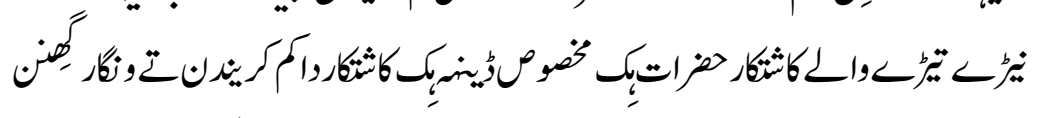

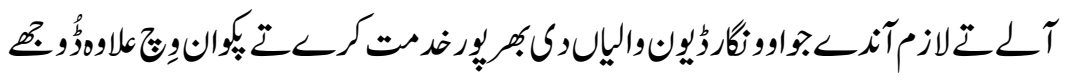

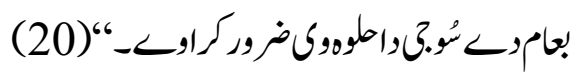

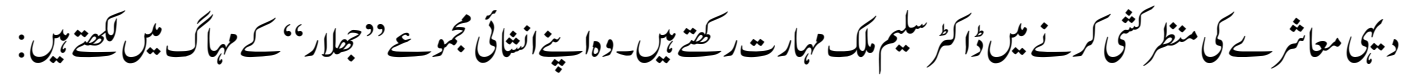

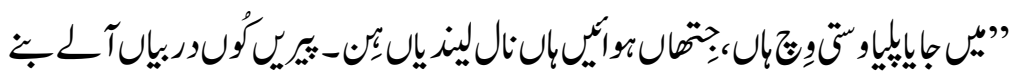

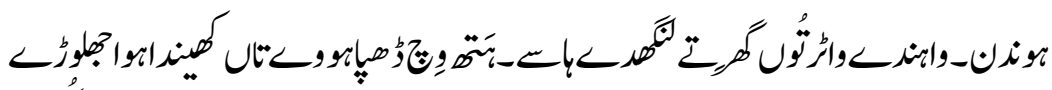

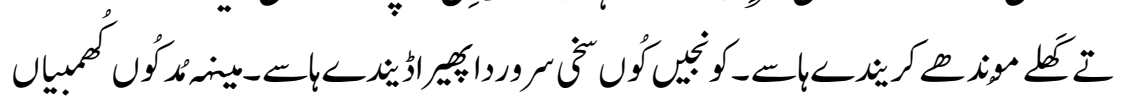

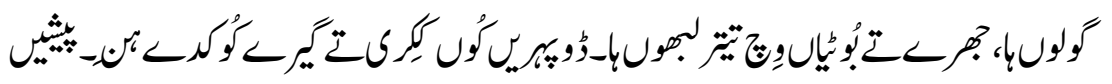

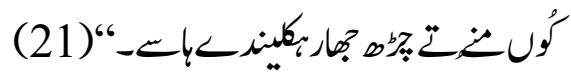




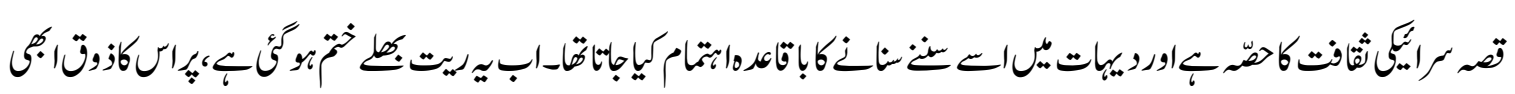

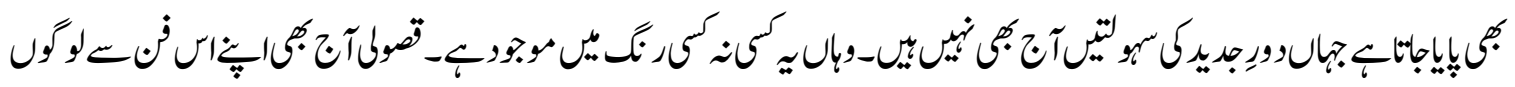

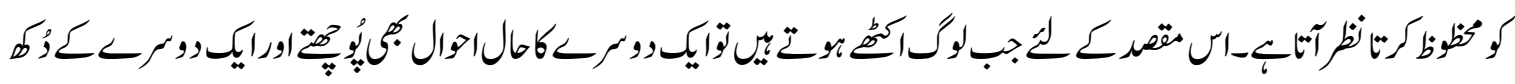

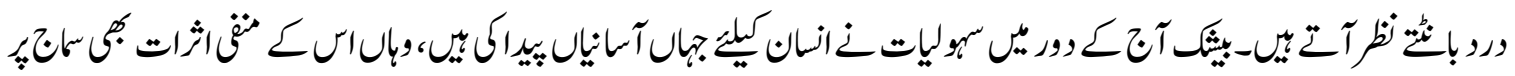

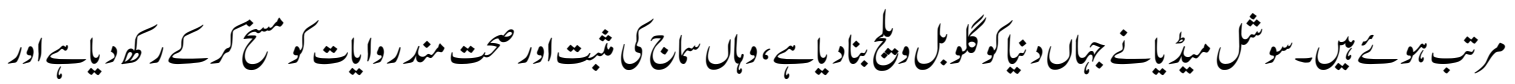

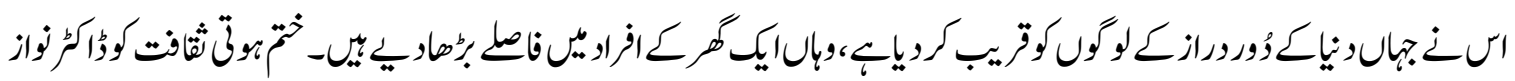

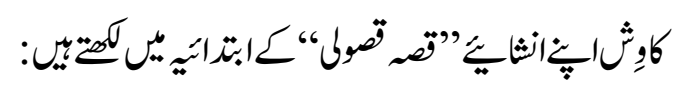

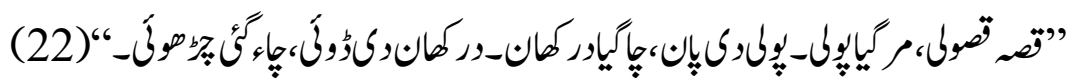

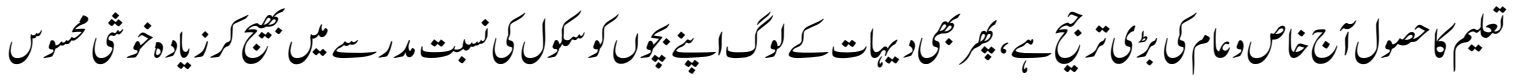

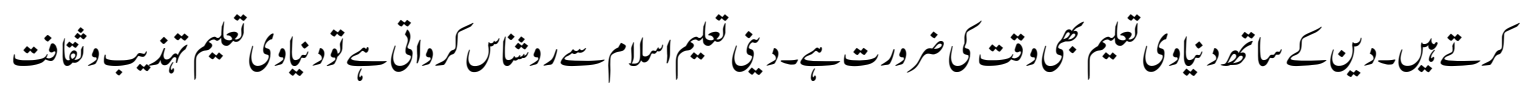

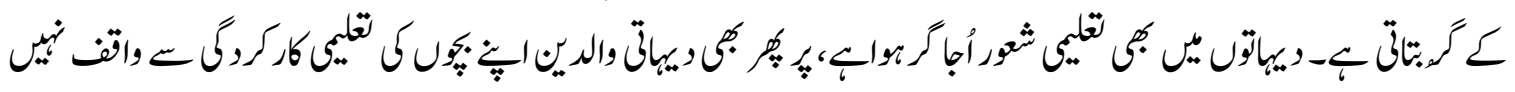

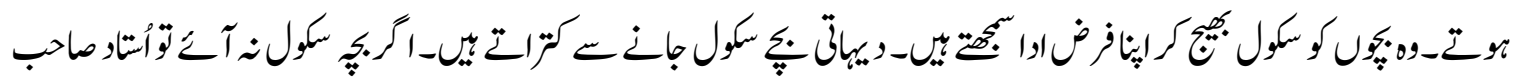

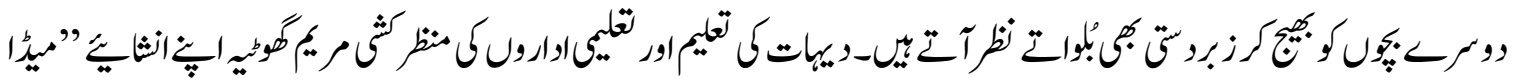

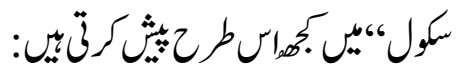

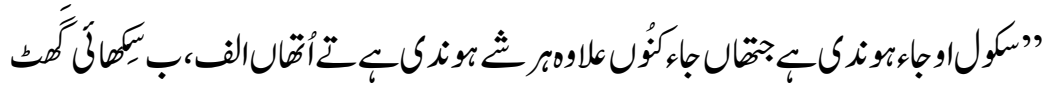

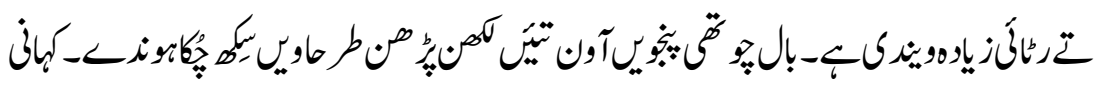

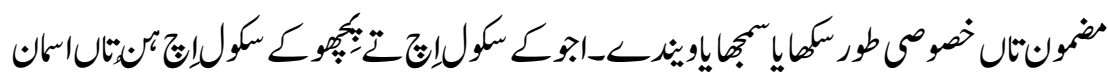

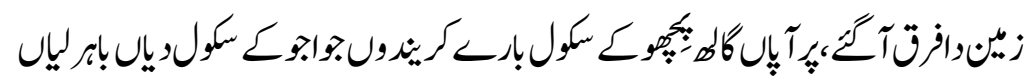

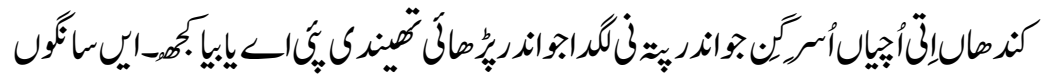

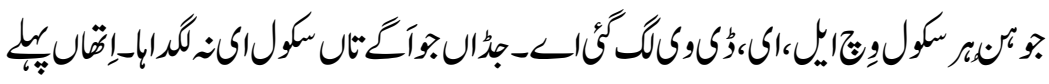

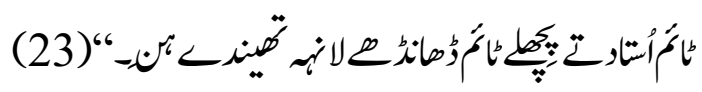

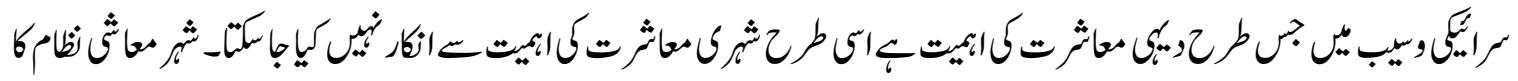

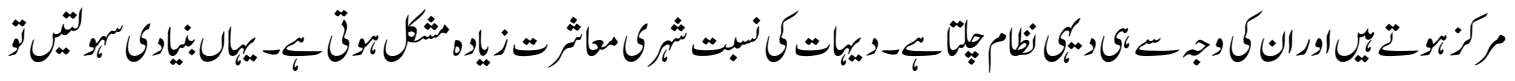




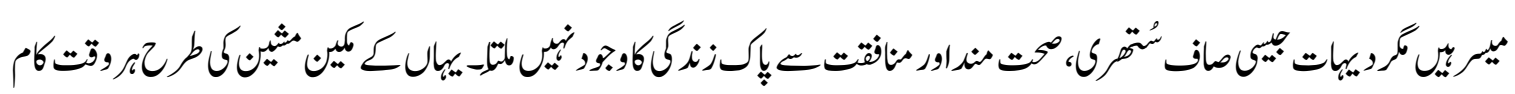

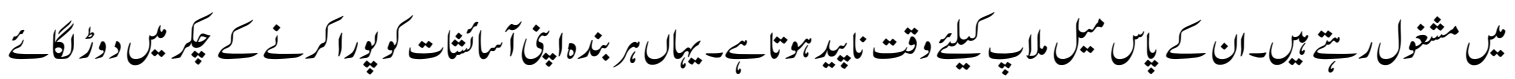

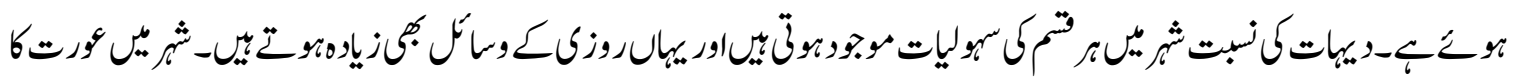

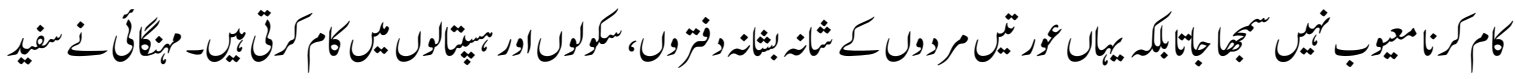

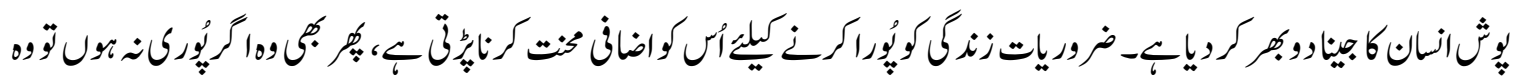

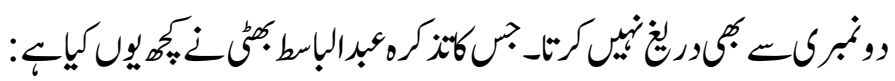

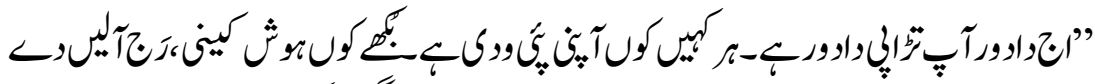

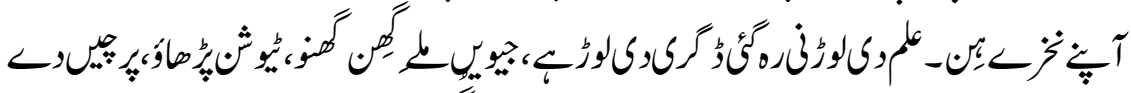

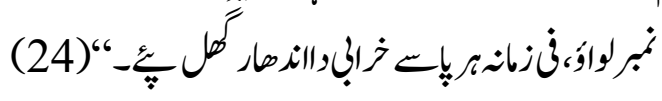

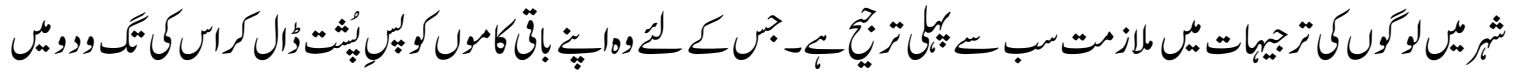

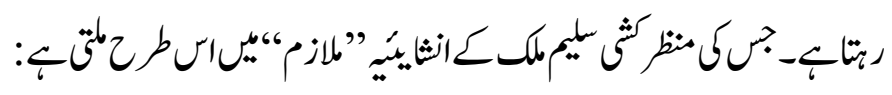

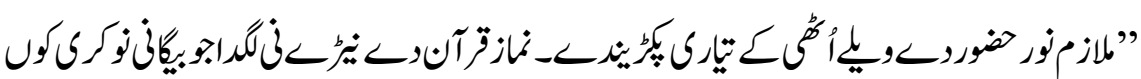

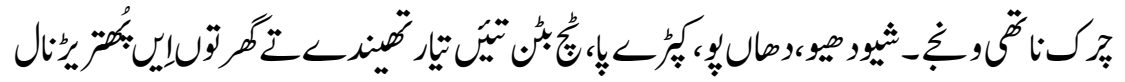

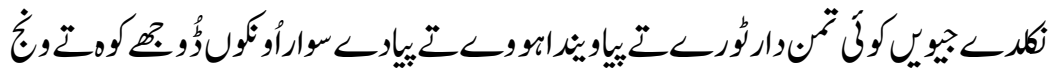

$$
\text { رك+_"(25) }
$$

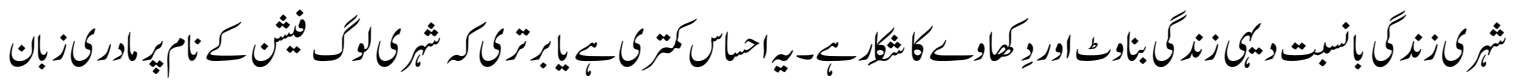

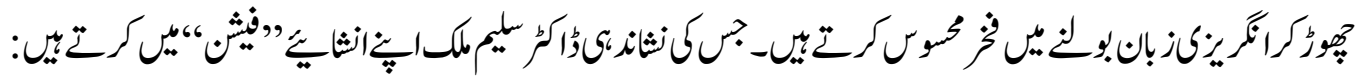

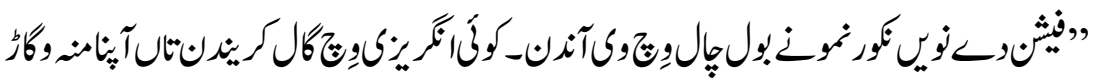

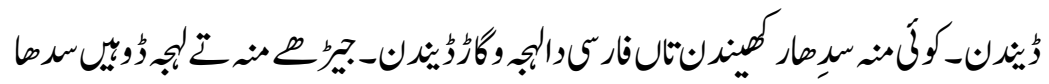

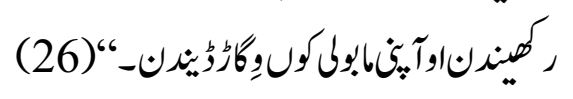

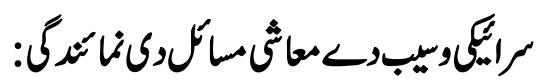

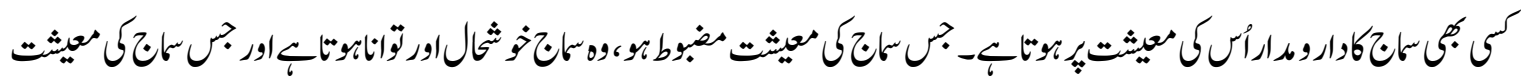

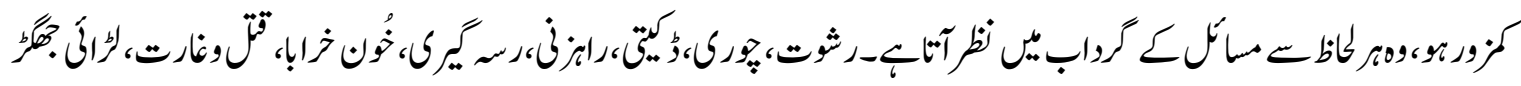

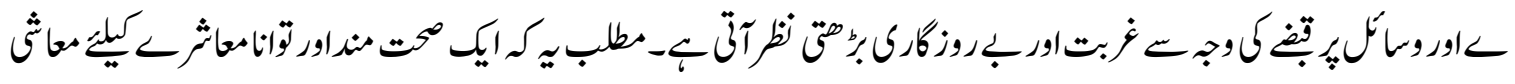




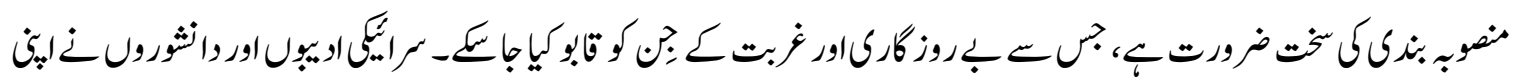

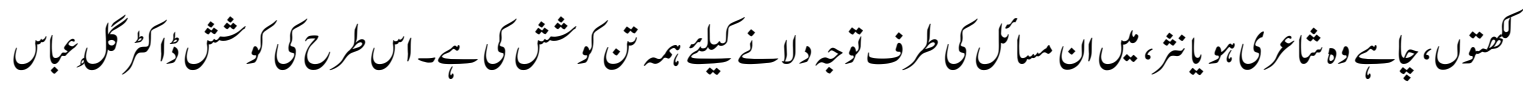

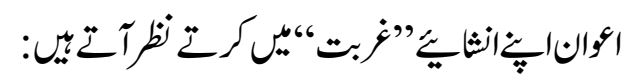

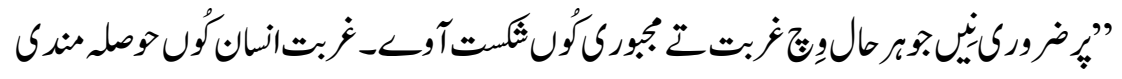

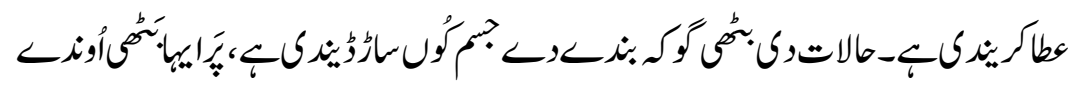

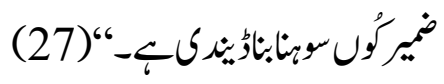

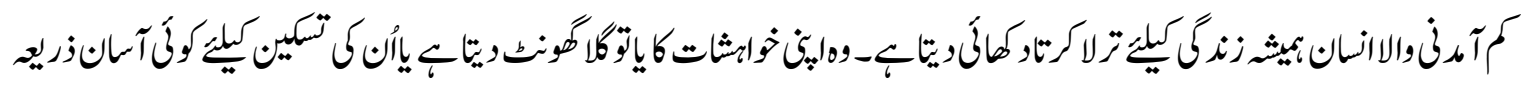

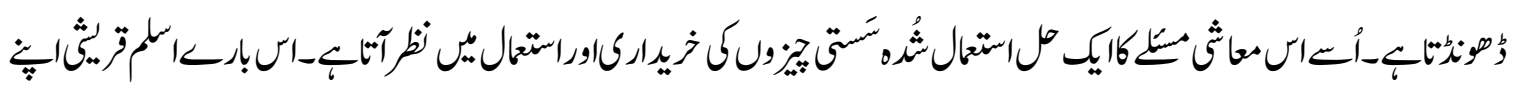

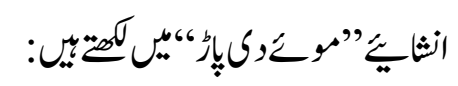

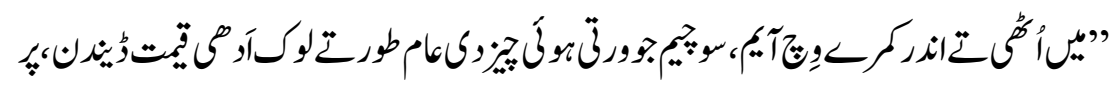

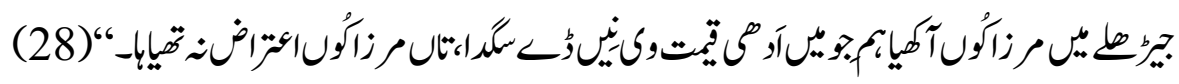

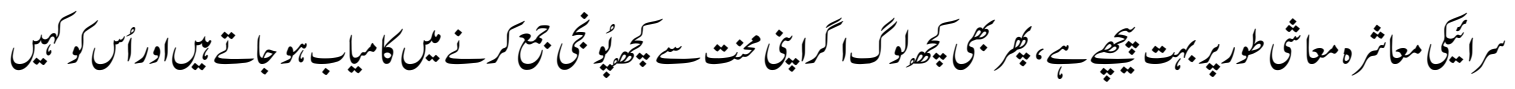

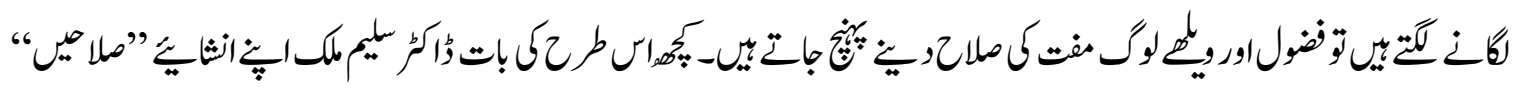

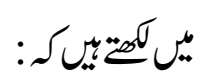

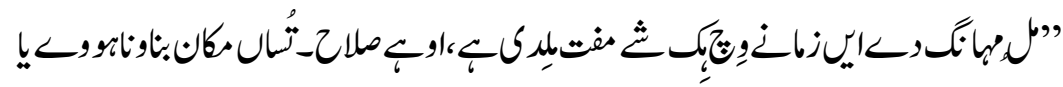

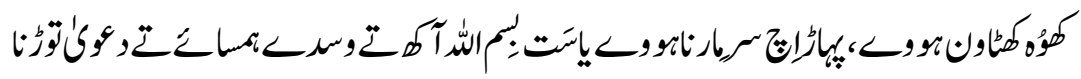

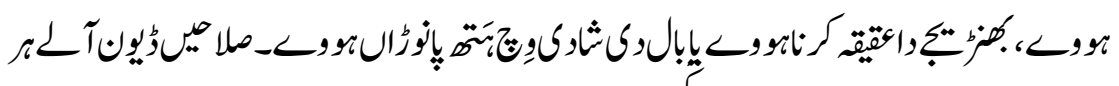

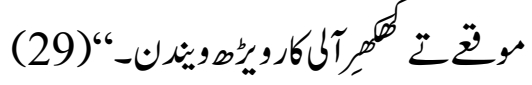

6م.

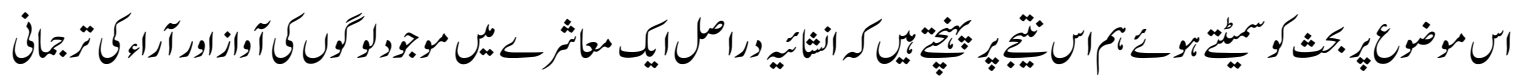

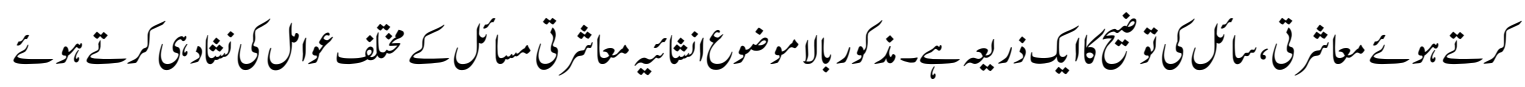

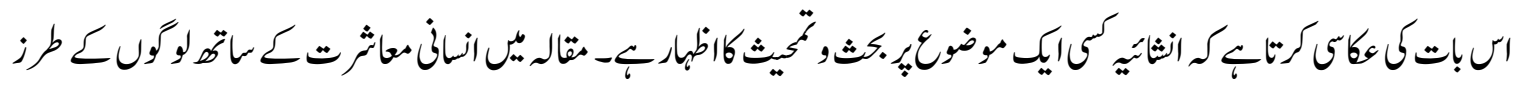

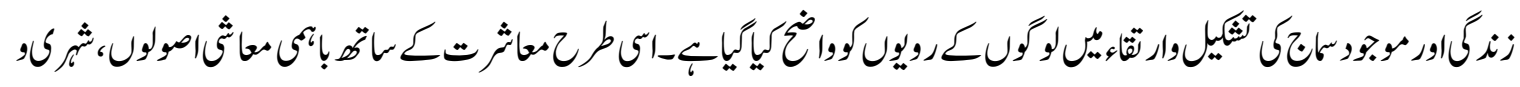

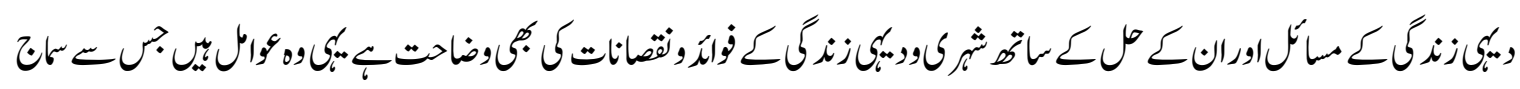




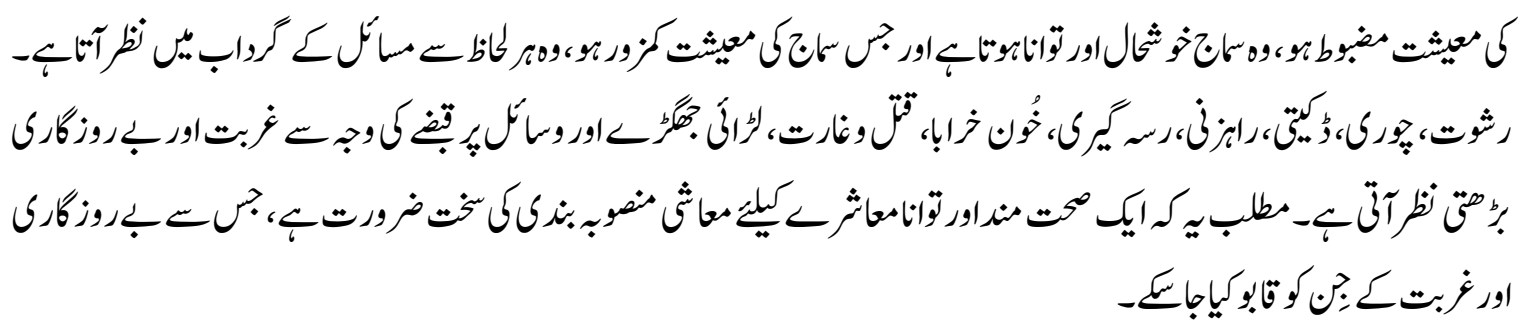

\section{References}

1- Shafiq Ahmed, Doctor, Roshan Ara Rao (Editor), Intikhab Insha'Allah Number (First Time), Department of Confessions, Bahawalpur, Islamia University of Bahawalpur, P.80

2- Tahir Tonsoi, Doctor, Satire, Humor, History, Criticism, Election, Lahore, Milestone Publications, P.15

3- Saleem Akhtar, Doctor, Insha'i Foundation, Lahore, Milestone Publications, P.114

4- Shaukat Mughal, Bataan Baturian, Multan, Jhuk Publishers, P.08

5- Mukhtar Ali Shah, Tradition of Insha'iyyah and Seraiki Insha'iyyah, including Quarterly Seraiki Bahawalpur, Issue No. 2, Volume No. 2, April to June, P.24

6- Mukhtar Ali Shah, Tradition of Insha'iyyah and Seraiki Insha'iyyah, including Quarterly Seraiki Bahawalpur, Issue No. 2, Volume No. 2, April to June, P.25

7- Saleem Akhtar, Doctor, Insha'i Foundation, Lahore, Milestone Publications, p.77

8- Saleem Akhtar, Doctor, Insha'i Foundation, Lahore, Milestone Publications, p.33

9- Gul Abbas Awan, Doctor, Sojhla, Multan, Jhuk Publishers, p.56

10- Saleem Malik, Doctor, Jhalar, Bahawalpur, Seraiki Literary Council, P.47

11- Aslam Mitla, Waseeb Rang, Khanewal, Mitla Publications, P.110

12- Abul Bashar Jilani, Syed, Bukh Da Makhol, Sadiqabad, Mubarak Urdu Library, P.30

13-Saleem Malik, Doctor, Jhalar, Bahawalpur, Seraiki Literary Council, P.84

14- Ahmed Saeed Rana, Sardar, Til Waseeb, Multan, Reza Mulla Printing Press, P.20

15- Nawaz Kaush, Doctor, Partaan, Bahawalpur, Cholistan Academic Literary Forum, P.34

16- Maryam Ghotia, Eye, Bahawalpur, Institute of Language and Literature Research, Bahawalpur, P.68

17- Sardar Hamad Saeed, Rana, Asun Pasoon, Darabpur (Jalalpur Pirwala), p.12

18- Bhapla, Malik Iqbal Hassan, Baloo, Jhuk Publishers, Multan, p.22

19- Abdul Basit Bhatti, Olandaran, Multan, Multan Institute of Policy and Research, P.62

20- Aslam Mitla, Seraiki Insha'i, Seraiki Publications, Muzaffargarh, p.18

21- Saleem Malik, Jhalar, Seraiki Literary Council, Bahawalpur, p.1

22-Nawaz Kaush, Partaan, Bahawalpur, Cholistan Literary Forum, p.57

23- Maryam Ghotia, Eye, Bahawalpur, Institute of Language and Literature Research, p.94

24- Dahir, Altaf Khan (Editor), Abdul Basit Bhatti Writings and Narwar, Multan, Jhook Publishers, p.366

25- Saleem Malik, Doctor, Jhalar, Bahawalpur, Seraiki Literary Council, p.68

26- Saleem Malik, Doctor, Jhalar, Bahawalpur, Seraiki Literary Council, p.75

27- Dr. Gul Abbas Awan, Sojhla, Multan, Jhuk Publishers, p.29

28- Aslam Qureshi, Katkaliyan, Bahawalpur, Seraiki Literary Council, p.85

29-Saleem Malik, Jhalar, Bahawalpur, Seraiki Literary Council,p.9 